\title{
Temporal Bell-type inequalities for two-level Rydberg atoms coupled to a high- $Q$ resonator
}

\author{
Susana F. Huelga, ${ }^{1}$ Trevor W. Marshall, ${ }^{2}$ and Emilio Santos ${ }^{3}$ \\ ${ }^{1}$ Departamento de Física, Universidad de Oviedo, 33007 Oviedo, Spain \\ ${ }^{2}$ Department of Mathematics, University of Manchester, Manchester M13 9PL, United Kingdom \\ ${ }^{3}$ Departamento de Física Moderna, Universidad de Cantabria, 39005 Santander, Spain
}

(Received 23 January 1996)

\begin{abstract}
Following the strategy of showing specific quantum effects by means of the violation of a classical inequality, a pair of Bell-type inequalities is derived on the basis of certain additional assumptions, whose plausibility is discussed in detail. Such inequalities are violated by the quantum mechanical predictions for the interaction of a two-level Rydberg atom with a single mode sustained by a high- $Q$ resonator. The experimental conditions required in order to show the existence of forbidden values, according to a hidden variables formalism, in a real experiment are analyzed for various initial field statistics. In particular, the revival dynamics expected for the interaction with a coherent field leads to classically forbidden values, which would indicate a purely quantum effect. [S1050-2947(96)04108-X]

PACS number(s): 03.65.Bz, 32.80.- t
\end{abstract}

\section{INTRODUCTION}

The aim of this paper is to extend our previous work [1] on temporal Bell inequalities and its possible experimental testing. In particular, we will analyze thoroughly the role of the additional assumptions required in order to formulate testable inequalities. As is well known, recent developments in cavity quantum electrodynamics and the generation and manipulation of atoms in highly excited states have provided situations where the most puzzling effects predicted by the quantum formalism might be observable. We refer to the well known paradoxes exemplified by Einstein, Podolsky, and Rosen [2] and Schrödinger [3], which address, respectively, the possible existence of nonlocal correlations and the possibility of producing a system in a coherent superposition of macroscopically distinguishable states.

As far as nonlocal correlations are concerned, the work by Bell [4] indicated how to make the transition from Gedanken to real experiments by deriving an inequality to be fulfilled by any local realist theory and which is violated by the quantum mechanical predictions in certain situations. However, none of the empirical tests performed until now (see Refs. [5,6] for reviews) can be considered uncontroversial [7]. Recently, Phoenix and Barnett [8] and independently Cirac and Zoller [9] have proposed a procedure for generating singletlike entangled states of two two-level atoms that have traversed successively an initially empty resonator, which might circumvent the loopholes remaining after the photon polarization correlation experiments. In addition, the proposed scheme could be generalized to produce entangled states involving more than two atoms and, hence, suitable for the one-shot experiment envisaged by Greenberger, Horne, and Zeilinger [10]. However, the situations considered are, for the moment, highly idealized, and a more detailed analysis is necessary before we can say whether such a proposal really provides a loophole-free test of the Bell inequality.

With respect to the so-called Schrödinger cat paradox, the problem can be formalized in a similar way by introducing inequalities to test for the existence of superpositions, distinguishable from statistical mixtures, of macroscopic states.
Inequalities of this kind were derived by Leggett and Garg from the premises of macroscopic realism and noninvasive measurability [11]. Because they involve measurements at different times on a single system, such inequalities are often referred to in the literature as temporal Bell inequalities. Some proposals for their empirical testing, using semiconducting quantum interference device techniques, have been made in recent years [11-13] but the extreme difficulty of experiments with truly macroscopic systems, as well as the difficulty concerning the plausibility of the noninvasive measurement assumptions, has made it impossible to draw any clear conclusion, and no empirical violations of the temporal Bell inequalities have been reported so far. (The current state of the art is reviewed in Ref. [14].)

A different approach to the problem is currently in progress at the Ecole Normale in Paris [15]. Nonclassical fields are generated in a high- $Q$ cavity containing a coherent field that is in interaction with a two-level atom prepared in a superposition of two Rydberg states of different energy. If the interaction is nonresonant, the field can be put into a cat state, namely, a superposition of two coherent states with opposite phase. The existence of this state would be demonstrated by studying the transitions of a second Rydberg atom. If the cavity contains a field describable in terms of a statistical mixture, the probability of detecting the atom in state $e$ or $g$ is $1 / 2$ each. However, if the field is in a cat state, the probe should be detected always in the same state as the initial atom. Thus, the conditional probability of detecting both atoms in the same state is expected to change continuously from 1 to $1 / 2$ as the time delay $t_{d}$ between the two detections varies from $t_{d} \ll \tau / \bar{n}$ to $t_{d} \gg \tau / \bar{n}$, where $\tau$ is the cavity relaxation time and $\bar{n}$ is the average number of photons in the coherent field.

It may appear that a similar scheme could be used in order to test a temporal Bell inequality, using sucessive atoms for probing the state of the field at different times. However, the dynamic evolution of the cat state does not display oscillatory behavior between the two macroscopically different states $|\alpha\rangle$ and $|-\alpha\rangle$, as would be necessary for the LeggettGarg inequalities to be violated. 
This paper addresses the Schrödinger problem at an intermediate level. We shall put forward arguments, in the context of a realist description, demanding that the systems involved exhibit a temporal evolution consisting of transitions between two allowed states, even though they cannot be considered as truly macroscopic. The paper is organized as follows: We first derive Bell-type inequalities in the framework of a very general hidden variables formalism for timedependent processes. As we shall show in Sec. II C, the derivation of testable inequalities requires that we introduce certain supplementary assumptions whose plausibility we discuss in detail in this and the following section. On this basis a pair of independent inequalities for the statistical predictions of a realist theory of a two-level stochastic process can be obtained. In particular, one of the inequalities obtained supersedes an inequality stated in our previous work [1]. Secondly, we propose a specific situation where violations of such inequalities by the quantum mechanical predictions might be observed, namely, a two-level Rydberg atom initially prepared in its excited state interacting with a single mode in a high- $Q$ resonator. In Sec. II D we compare our proposal with the Leggett-Garg approach for testing macroscopic quantum coherence.

Section III is devoted to the analysis of the quantum mechanical predictions with two different initial fields. We shall see that, when the cavity contains a thermal field, the departures from the realist bounds increase with decreasing temperature, being maximum in the ideal case of the cavity at $T=0 \mathrm{~K}$, a fact that we have already discussed [1]. A comparison with presently available experimental data is presented in Sec. III B. In addition, when the cavity contains initially a coherent field, two domains leading to classically forbidden values appear, corresponding to very short and very long interaction times, the latter covering the revival region. This indicates that the revival dynamics may be interpreted as a purely quantum effect. Finally, Sec. IV draws the conclusions that may be inferred from the proposed tests.

\section{THE HIDDEN VARIABLES APPROACH FOR TIME-DEPENDENT PROCESSES}

\section{A. Bell's formulation of hidden variables theories}

As is well known, a hidden variables theory starts from the basis that a more complete specification of the state of a given system than that provided by the quantum mechanical wave function is possible. This idea was advocated, among others, by Einstein [16]. Following Bell's formulation [4], let this more complete description be effected by means of a parameter or set of parameters $\lambda$. In our case, $\lambda$ should specify the state of the composite system atom plus cavity. The core of a hidden variables theory is the assumption that systems that are identical according to quantum mechanics may be nonidentical. Then, let $\rho(\lambda)$ be the normalized probability density characterizing an ensemble of systems resulting from an identical preparation technique. The probability of detecting the atom in the state $e$ at time $t$ would be given by

$$
P_{e}(t)=\int d \lambda \rho(\lambda) M_{e}(\lambda, t),
$$

where $M_{e}(\lambda, t)$ is a dichotomic function with values 0 or 1 and involves, somehow, the evolution of the system. This function would exhibit, in most cases, a very complicated dependence on the initial conditions, but the essential point is that, once it is specified, it can be stated whether a definite event will or will not take place at a given time. Note that this deterministic approach allows us to classify, at any time, the set of possible $\lambda$ into two subsets corresponding to the values that give $M_{e}(\lambda, t)=1$ and $M_{e}(\lambda, t)=0$, respectively. This can be interpreted by saying that, at any time, the atom $i s$ either in state $e$ or in state $g$.

For the sake of clarity let us consider an example extracted from classical physics. Imagine that we throw coins with the aid of a certain machine. According to classical physics a complete description of the state of the coin requires the specification of twelve parameters (the set $\lambda$ in the general approach). If we know exactly these values at the initial time, then it should be possible in principle to predict whether the coin will be in state head or tail after the collision with the floor. That is, it would be possible to construct a function of the initial conditions $\lambda$ and the time $t$, dichotomic but with a complicated dependence on these variables, such that, given $\lambda$ and $t$, we can state the output of the collision with the floor. There is no doubt that, somehow, such a function contains the temporal evolution of the system. The second assumption, in a classical description, concerns the fact that, once the mechanism for throwing the coin is specified, the initial values for different coins will not be, in general, identical. Hence, ensemble probabilities can be evaluated only after introducing the probability distribution over $\lambda$. In other words, the essential ingredient of a hidden variables formalism is the existence of the functions $\rho(\lambda)$, positive and normalized, and $M(\lambda, t) \in\{0,1\}$. This suffices for determining the probabilities of all possible different events after the interaction with a given measuring apparatus. For instance, the joint probability for the atom to be (detected) in level $e$ at times $t_{1}$ and $t_{2}$ would be calculated as

$$
P_{e e}\left(t_{1}, t_{2}\right)=\int d \lambda \rho(\lambda) M_{e}\left(\lambda, t_{1}\right) M_{e}\left(\lambda, t_{2}\right) .
$$

The single time probabilities such as (1), two-time probabilities such as (2), three-time probabilities defined in an analogous way, etc., fully define a dichotomic stochastic process with values 0 and 1 . It could be argued that probabilities such as (2) are unphysical because they cannot be measured, in spite of the fact that they have all the mathematical properties for a quantity to be a probability. In any case, and this will be clear later, their mere existence leads to physical constraints in terms of certain inequalities to be fulfilled by the statistical predictions of the hidden variables formalism.

At this point, it is worthwhile to make two additional comments. (i) It should be noted that different values of $\lambda$ can yield, at a given time, the same result, $e$ or $g$, for the state of the atom after the interaction with a suitable two channel detection apparatus. In other words, atoms detected in the same state do not necessarily correspond, in a realist representation, to ensembles of subquantum states with the same probability distribution over the hidden variables. Indeed, if this were not the case, interference effects could not be explained by means of a hidden variables approach. (ii) In the light of the preceding comment, the widespread assertion 
that according to a hidden variables formalism, a measurement simply reveals a pre-existing value seems to be, in a certain sense, misleading. Invoking again the classical process of throwing a coin, one can ask whether it makes any sense to say that the coin is in state head or tail before the collision with the floor has taken place. However, objective properties can be assigned to the system at any time, for instance, the velocity and position of its center of mass.

In the general approach that we are trying to present here, realism enters through the assumption that, given a preparation technique, the state of the system can be characterized in terms of certain $\lambda$ and no restriction is made about the possible complexity of this parameter. For instance, as stated above, $\lambda$ can be viewed as the initial state of the system, which suffices to determine the probability of different outcomes after the interaction with a given apparatus. If the theory is deterministic, then, given $\lambda$ and $t$, it should be possible to determine with certainty which outcome will take place.

It is also possible to introduce a probabilistic approach, where only the probabilities for the different outcomes are predicted. In that case, $M_{e}(\lambda, t)$ in Eq. (1) should be replaced by a function $P_{e}(\lambda, t)$, taking a real value between 0 and 1 . In the example of the coin we may assume that the initial conditions do not completely determine the result, head or tail, due to uncontrollable perturbations such as, for example, the wind.

Note that in this case it is not possible to divide the set of $\lambda$ into two subsets at any time corresponding, respectively, to the states $e$ and $g$. Nevertheless, the probabilities (1), (2), etc. can be defined, and the evolution can be represented as a dichotomic stochastic process. In any case, the point is that the system has objective properties, represented by the global parameter $\lambda$ before, and independently of, whether a measurement is performed or not. These properties, which may be identified with the elements of reality introduced by Einstein, Podolsky, and Rosen should be distinguished from the experimental quantities, the outcomes $e$ and $g$ for the atomic state in our case. This topic has been extensively discussed in Ref. [17].

\section{B. Inequalities for dichotomic stochastic processes}

We have seen, in the preceding section, how a hidden variables formalism allows us to represent a time-dependent evolution as a stochastic process. In particular, it is easy to show that the two-time autocorrelation functions defined as

$$
K\left(t_{i}, t_{j}\right)=P_{e e}\left(t_{i}, t_{j}\right)-P_{e g}\left(t_{i}, t_{j}\right)+P_{g g}\left(t_{i}, t_{j}\right)-P_{g e}\left(t_{i}, t_{j}\right),
$$

where $i, j \quad(i=j)$ stands for $1,2,3$, are bounded by inequalities of the form

$$
K\left(t_{1}, t_{3}\right) \pm\left[K\left(t_{1}, t_{2}\right)+K\left(t_{2}, t_{3}\right)\right] \geqslant-1
$$

whose derivation was presented in [1].

Taking into account that $P_{e}\left(t_{1}\right)$ can be expressed as

$$
P_{e}\left(t_{1}\right)=P_{e e}\left(t_{1}, t_{2}\right)+P_{e g}\left(t_{1}, t_{2}\right)
$$

$$
P_{e}\left(t_{1}\right)=P_{e e}\left(t_{1}, t_{3}\right)+P_{e g}\left(t_{1}, t_{3}\right)
$$

and the obvious relation

$$
P_{g g}\left(t_{1}, t_{2}\right)=1-P_{e e}\left(t_{1}, t_{2}\right)-P_{e g}\left(t_{1}, t_{2}\right)-P_{g e}\left(t_{1}, t_{2}\right),
$$

the autocorrelation function (3) for times $t_{1}$ and $t_{2}$ can be expressed as

$$
K\left(t_{1}, t_{2}\right)=1-2\left[P_{e}\left(t_{1}\right)+P_{e}\left(t_{2}\right)-2 P_{e e}\left(t_{1}, t_{2}\right)\right] .
$$

Now, in order to get an inequality in terms of directly testable quantities, let us introduce the conditional probabilities for the process, namely,

$$
Q_{e e}\left(t_{1}, t_{2}\right)=\frac{P_{e e}\left(t_{1}, t_{2}\right)}{P_{e}\left(t_{1}\right)} .
$$

For instance, $Q_{i j}\left(t_{1}, t_{2}\right)$ will denote the probability for the atom to be in the state $j$ (more precisely, to be found in state $j$ if it were measured) at time $t_{2}$ if it were in the state $i$ at time $t_{1}$. In terms of these quantities we can write the single time probabilities as follows:

$$
P_{e}\left(t_{2}\right)=P_{e}\left(t_{1}\right) Q_{e e}\left(t_{1}, t_{2}\right)+P_{g}\left(t_{1}\right) Q_{g e}\left(t_{1}, t_{2}\right) .
$$

Finally we consider the concrete experimental situation where the atoms have been initially prepared in the excited state. Then, considering the time sequence $t_{1}=0, t_{2}=t$, and $t_{3}=2 t$, and the initial condition

$$
P_{e}\left(t_{1}\right)=1
$$

the autocorrelation functions (8) can be writen as

$$
\begin{gathered}
K(0, t)=2 Q_{e e}(0, t)-1, \\
K(0,2 t)=2 Q_{e e}(0,2 t)-1, \\
K(t, 2 t)=1-2\left[Q_{e e}(0, t)+Q_{e e}(0,2 t)-2 Q_{e e}(0, t) Q_{e e}(t, 2 t)\right],
\end{gathered}
$$

taking account of relations (9) and (10) and condition (11). Then, the first inequality (4) yields

$$
Q_{e e}(0, t) Q_{e e}(t, 2 t) \geqslant 0,
$$

which is a trivial result. Similarly, the second inequality (4) can be rewritten in the form

$$
Q_{e e}(0, t) Q_{e e}(t, 2 t) \leqslant Q_{e e}(0,2 t),
$$

which is trivial as well. In fact, adding the positive quantity $Q_{e g}(0, t) Q_{g e}(t, 2 t)$ to the left side, we obtain the right one.

This is not a surprising result. Bell-type inequalities are always trivial in a classical approach, where the usual, Kolmogorovian, rules for probabilities apply. The problem is that, in both (15) and (16), the quantity $Q_{e e}(t, 2 t)$, which is not measurable, is involved. Note that $t$ corresponds to an intermediate interaction time between the injection of the

or as 
atom at $t=0$ and the exit from the cavity at a time $2 t .{ }^{1}$

It is then clear that the general hidden variables approach leads to inequalities that, as well as being trivial, are not amenable to experimental testing, due to the impossibility of performing measurements at intermediate times. To obtain testable inequalities we have to introduce some additional assumptions. A possible strategy for that will be discussed in the next section.

It should be emphasized that there is a clear difference between the above situation and that of measurements made on spatially separated systems; in the latter case testable inequalities can be obtained simply by requiring realism and locality. But even in that case the experimental conditions required to show divergences from the quantum-mechanical predictions are extremely demanding, and all experiments performed until now have had to introduce supplementary assumptions such as no enhancement. Only recently [18-20] have loophole-free tests for the Bell inequality been proposed, and none of them has yet been performed.

\section{Testable inequalities}

As previously stated in Sec. II A, a hidden variables representation of a pure quantum state is provided by certain probability distribution $\rho(\lambda)$. Given that we are dealing here with time-dependent processes, let us rewrite $\rho(\lambda)$ as $\rho(\lambda, 0)$, in order to emphasize that this stands for the probability distribution over the hidden variables at the initial time $t=0$. At a latter time $t$, the hidden variables have evolved from the initial value $\lambda$ at $t=0$ to certain $\mu$ whose value will depend on both the initial conditions and the elapsed time, $\mu=\mu(\lambda, t)$. Denoting by $\Lambda$ the set of all possible values of $\lambda$, this set can be divided into two disjoint subsets $\Lambda_{e}$ and $\Lambda_{g}$ such that if $\lambda \in \Lambda_{e}$, then $M_{e}(\lambda, 0)=1$ whereas $M_{e}(\lambda, 0)=0$ as long as $\lambda \in \Lambda_{g}$. In the same way, if $\mu(\lambda, t) \in \Lambda_{e}$ then $M_{e}(\mu(\lambda, t), 0) \equiv M_{e}(\lambda, t)=1$ and, if $\mu(\lambda, t) \in \Lambda_{g}$, it is $M_{e}(\mu(\lambda, t), 0)=0$. This can be generalized by writing ${ }^{2}$

$$
M_{e}\left(\mu\left(\lambda, t_{1}\right), t_{2}\right)=M_{e}\left(\lambda, t_{1}+t_{2}\right) .
$$

As far as the temporal evolution of the probability distribution is concerned, $\rho(\lambda, 0)$ induces at time $t$ a probability distribution over the hidden variables $\mu(\lambda, t)$ that we will denote as $\rho(\mu, t)$. If no additional assumption is imposed on either the hidden variables or the functional form of the corresponding probability density, it is not possible to derive testable Bell-type inequalities, as clearly shown in the preceding section. The next step in our procedure will consist of introducing certain supplementary hypotheses in order to avoid the need of measuring at intermediate times and hence to derive inequalities amenable for experimental testing.

\footnotetext{
${ }^{1}$ In the classical macroscopic domain, it is not always possible to measure at intermediate times (Leggett-Garg assumption of noninvasive measurability). This is, for example, the case in the example of the coin. Therefore, measurability is not the essential feature of classical physics, but rather the existence of the functions $\rho(\lambda)$ and $M_{e}(\lambda, t)\left[\right.$ or $\left.P_{e}(\lambda, t)\right]$.

${ }^{2}$ Note that $\mu\left(\lambda, t_{1}+t_{2}\right)=\mu\left(\mu\left(\lambda, t_{1}\right), t_{2}\right)$.
}

\section{Two-state hypothesis}

We will assume that, at any time, the probability distribution function can be decomposed in the form

$$
\rho(\mu, t)=w(t) \rho_{e}(\mu)+(1-w(t)) \rho_{g}(\mu),
$$

where $w$ is a time-dependent weight factor, $w(t) \in[0,1]$. The probability densities $\rho_{e}$ and $\rho_{g}$ are normalized and timeindependent and have support in $\Lambda_{e}$ and $\Lambda_{g}$, respectively. In other words, the state of the system at any time is supposed to be given by a statistical mixture of just two states, $e$ and $g$.

If we consider a definite experimental situation where the atoms are initially prepared in the upper level $e$, then $w(0)=1$ and $\rho(\lambda, 0)=\rho_{e}$. The assumption (18) leads us to set the identity

$$
\rho(\lambda, 0)=\rho_{e}(\lambda) .
$$

Let us analyze the meaning of this hypothesis, whose clear understanding is essential for setting both the scope of the testable inequalities that we will derive on its basis and the conclusions that may be inferred from its eventual violation in a real experiment. The idea is as follows: A set of atoms prepared in the Rydberg state $e$ interacts for a finite time with a microwave cavity tuned to the $e \rightarrow g$ transition frequency. Once the atoms exit the cavity, the atomic state can be checked by means of the interaction with a suitable twochannel apparatus, which provides two exhaustive and mutually exclusive outputs. We have assumed that a hidden variables representation of any pointer state is an ensemble of microstates with always the same probability distribution of hidden variables. Thus, for example, an atom emerging in the state $e$ has precisely the same distribution of microstates as has the incident atom, and this state is associated with a definite state of the cavity which also coincides with its initial state. This latter state also is associated with a definite distribution of microstates, and in this case we may identify the hidden variables as, for example, the amplitudes and phases of all the cavity modes. The same argument may be used to support the idea that an atom emerging in state $g$ is associated with a definite state of the cavity, which may be informally described as "the initial state plus one photon."

As previously stated in Sec. II A, this assumption is quite restrictive for a general (microscopic) object. Because some subtle points are involved, we postpone to the next section the discussion about its plausibility for the system under consideration. For that it will be useful to compare our proposal with the Leggett and Garg approach to the Schrödinger cat problem.

Once the additional assumption (19) is introduced, it yields immediately, taking into account the definitions (1), (2), and the rule (17), the translational invariance (stationarity) of any conditional probability. That is,

$$
Q_{i j}\left(t_{i}, t_{j}\right)=Q_{i j}\left(t_{i}+\tau, t_{j}+\tau\right)
$$

for any value of $\tau$ and any $t_{i}, t_{j}$. In particular

$$
Q_{e e}(t, 2 t)=Q_{e e}(0, t) .
$$

In these conditions inequality (16) yields 


$$
Q_{e e}(0,2 t) \geqslant Q_{e e}^{2}(0, t),
$$

which is amenable to experimental testing by a two-shot experiment in which the number of atoms that remain in the excited state are measured after two different interaction times $t$ and $2 t$. The interaction time can be controlled, for instance, by means of a suitable velocity selection.

\section{Symmetry assumption}

In addition, admitting as a second supplementary assumption the symmetry between the states $e$ and $g,{ }^{3}$ that is

$$
Q_{e g}\left(t_{1}, t_{2}\right)=Q_{g e}\left(t_{1}, t_{2}\right),
$$

which implies that

$$
Q_{e e}\left(t_{1}, t_{2}\right)=Q_{g g}\left(t_{1}, t_{2}\right)
$$

[Alternatively, we could postulate that $Q_{e e}\left(t_{1}, t_{2}\right)$ $=Q_{g g}\left(t_{1}, t_{2}\right)$, and then deduce that $Q_{e g}\left(t_{1}, t_{2}\right)$ $=Q_{g e}\left(t_{1}, t_{2}\right)$.] We will obtain that any two-time autocorrelation function will depend only on the time difference. This property will result into two new Bell-type inequalities. In fact, the autocorrelation function (3) can be rewritten in terms of conditional probabilities as

$$
\begin{aligned}
K\left(t_{1}, t_{2}\right)= & P_{e}\left(t_{1}\right)\left[Q_{e e}\left(t_{1}, t_{2}\right)-Q_{e g}\left(t_{1}, t_{2}\right)\right]+P_{g}\left(t_{1}\right) \\
& \times\left[Q_{g g}\left(t_{1}, t_{2}\right)-Q_{g e}\left(t_{1}, t_{2}\right)\right],
\end{aligned}
$$

Taking into account that $P_{e}+P_{g}=1$ and the obvious relations $Q_{e g}=1-Q_{e e}$ and $Q_{g e}=1-Q_{g g}$, we get

$$
\begin{aligned}
K\left(t_{1}, t_{2}\right)= & P_{e}\left(t_{1}\right)\left[2 Q_{e e}\left(t_{1}, t_{2}\right)-1\right]+\left(1-P_{e}\left(t_{1}\right)\right) \\
& \times\left[2 Q_{g g}\left(t_{1}, t_{2}\right)-1\right] .
\end{aligned}
$$

Then, we can write on the basis of the symmetry assumption that

$$
K\left(t_{1}, t_{2}\right)=2 Q_{e e}\left(t_{1}, t_{2}\right)-1
$$

and the stationarity of conditional probabilities yields the same property for autocorrelation functions, that is

$$
K\left(t_{1}, t_{2}\right)=K\left(0, t_{2}-t_{1}\right) .
$$

The reciprocal is not true unless one admits that the single probabilities $P_{e}$ and $P_{g}$ are time independent. With this result, inequalities (4) lead to the testable forms

$$
\begin{gathered}
Q_{e e}(0,2 t)+2 Q_{e e}(0, t) \geqslant 1 \\
Q_{e e}(0,2 t)-2 Q_{e e}(0, t) \geqslant-1
\end{gathered}
$$

It is easy to see that inequality (22) implies (30). Starting from the obvious relation

$$
\left[1-Q_{e e}(0, t)\right]^{2}=Q_{e e}^{2}(0, t)-2 Q_{e e}(0, t)+1 \geqslant 0,
$$

\footnotetext{
${ }^{3}$ Note that the quantum formalism satisfies this requirement. On the other hand, it is not necessary to introduce this assumption for deriving the inequality (22).
}

the inequality (22) leads to

$$
Q_{e e}(0,2 t) \geqslant Q_{e e}^{2}(0, t) \geqslant 2 Q_{e e}(0, t)-1,
$$

and this is precisely the inequality (30). However, the inequality (29) is independent of (22) as can be easily verified. As a consequence, it is worthwhile to test the inequalities (22) and (29), while the inequality (30) is superseded by (22). We will discuss in Sec. IV the conclusions that might be drawn if such inequalities are violated in a real test.

\section{Comparison with Leggett-Garg proposal for testing macroscopic quantum coherence}

The Schrödinger cat paradox shows that the extrapolation of the quantum formalism to the macroscopic level leads to superpositions of distinguishable states that do not seem to exist in nature. The absence of truly macroscopic systems in superposition states is currently explained by the decoherence mechanism [21]. The approach of Leggett and Garg [11] tries to formulate the problem in a way suitable for experimental testing. On the basis of two hypotheses, namely, macroscopic realism (MR) and noninvasive measurability (NIM), at first sight very natural in the framework of a realist description of the macrolevel, they derive an inequality, formally equivalent to the familiar one of Clauser, et al. [22], that is

$$
\left|K\left(t_{1}, t_{2}\right)+K\left(t_{2}, t_{3}\right)+K\left(t_{3}, t_{4}\right)-K\left(t_{1}, t_{4}\right)\right| \leqslant 2,
$$

which is violated, at least for idealized systems, by the quantum mechanical predictions. At this point it is worth making two comments:

(i) The assumption of MR was stated as follows: If $a$ macroscopic system is, whenever observed, found to be in one of two macroscopically distinguishable states, then one can assign to it the property of actually being in one of these two states at any time. It should be noted that this assumption does not strictly hold for any classical system (let us think of the example of the coin advocated in Sec. II A, for instance). We can say, however, that it holds in a broad sense, as long as it is possible to determine the state of the system (the values of $\lambda$ ) and from that it is possible in principle to calculate the values of $M_{e}(\lambda, t)$ at any time. In a broad sense the MR assumption is incorporated to our description by means of the decomposition of the set of states $\lambda$ as $\Lambda=\Lambda_{e} \cup \Lambda_{g} \cdot{ }^{4}$

(ii) The assumption of NIM ensures the possibility of performing measurements with an arbitrarily small disturbance in the subsequent dynamics. This assumption is necessary in order to obtain any inequality capable of contradicting the quantum mechanical predictions. Once it is admitted, any two-time autocorrelation function depends only on the time difference and the resulting testable inequality involves two measuments at times $t$ and $2 t$. It should be noted that the assumption of NIM does not hold for all classical systems. For instance, it fails in the example of the coin, where a

\footnotetext{
${ }^{4}$ In any case, we must stress that this form would not be feasible in a hidden variables formalism admitting an essential indeterminism, where functions $M_{e}(\lambda, t)$ should be replaced by $P_{e}(\lambda, t)$.
} 
measurement of the dichotomic observable head or tail involves stopping the coin. But it holds in a certain sense, given that noninvasive procedures can be envisaged (a sequence of stroboscopic photographs in the case of the coin, for instance) to detect the hidden variables, whence the result of the measurement may be predicted.

In the experimental situation proposed in this paper, any measurement is obviously invasive, actually it is destructive. In addition, the system involved is not macroscopic. However, it may be argued that it exhibits certain features resembling those of a two-level macroscopic system. In particular, (i) due to the high principal quantum number $(n \sim 60)$, the system really lies in the semiclassical region. (ii) the linear dimensions of these atoms can be considered as mesoscopic; in fact, its size is comparable with the size of large biomolecules.

Then, the NIM in the Leggett-Garg approach is replaced in our case by the assumption (18), which constitutes a sufficient condition for precluding the existence of superposition states. It could be argued that a less naive hidden variables formalism might admit, in addition to two states and statististical mixtures of them, probability densities $\rho(\lambda)$ not obeying (18). This would correspond to the quantum mechanical superposition states. However, we cannot formulate any approach of this kind yielding testable inequalities. On the other hand, Eq. (18) allows us to maintain the analogy with the quantum formalism where the state $|e\rangle$ is always the same and should correspond to $\rho_{e}(\lambda)$, and the same applies for the state $|g\rangle$. The point is to study whether the quantum representation $c_{e}|e\rangle+c_{g}|g\rangle$ can be substituted by $w \rho_{e}+(1-w) \rho_{g}$, where $w=\left|c_{e}\right|^{2}$, with the obvious relation $\left|c_{e}\right|^{2}+\left|c_{g}\right|^{2}=1$.

Finally, we remark that our formulation presents some advantages compared to Leggett-Garg. As far as the experimental testing is concerned, the quadrilateral Leggett-Garg form based on NIM demands two sequential measurements at times $t$ and $3 t$

$$
|3 K(0, t)-K(0,3 t)| \leqslant 2
$$

while the inequalities that we have derived requires a second measurement at a time $2 t$, and is therefore less sensitive to dissipative effects. In addition, as showed in Fig. 1, the pair of inequalities that we have obtained cover more extended forbidden regions.

\section{QUANTUM-MECHANICAL PREDICTIONS}

Let us consider a single-mode resonator into which excited two-level atoms are injected at a rate low enough that the time interval between successive atoms is greater than the cavity damping time $\tau$. This requirement ensures that the same field repeats when each atom enters the cavity. In addition, whenever the atom-field interaction time is much shorter than $\tau$, the relaxation of the cavity mode can be ignored while an atom is inside the resonator, the coupled field-atom system being described by the Jaynes-Cummings Hamiltonian $[23,24]$. The evolution of the field during the intervals between successive atoms is governed by the master equation of a harmonic oscillator coupled to a thermal bath [25].

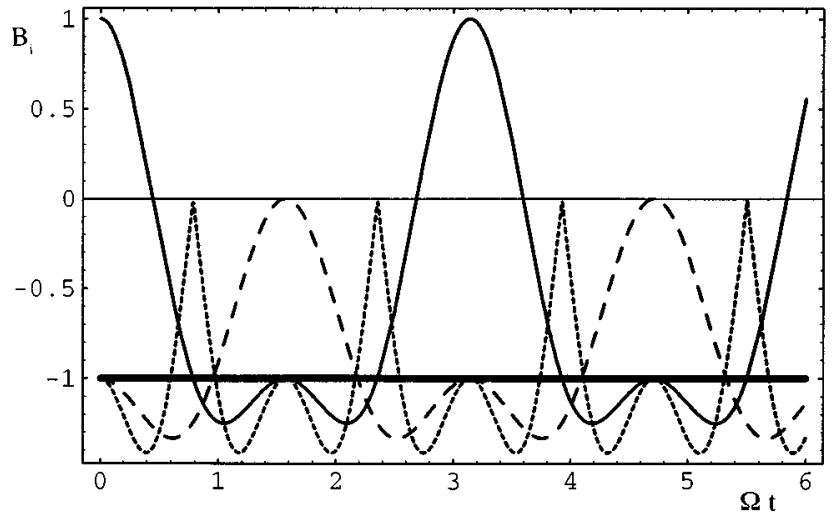

FIG. 1. Functions $B_{1}$ and $B_{2}$ (dashed curve) vs $\Omega t$ for the ideal case $T=0 \mathrm{~K}$. Violations of the Bell-type inequalities occur whenever either curve goes below -1 . The dotted curve represents the function obtained in the Leggett-Garg approach, Eq. (34), rewritten in a form useful for comparison with the $B_{i}$.

\section{A. Ideal case. Interaction with a Fock state}

Provided that the atom is initially in its upper state $e$ and that the cavity contains exactly $n$ photons, the pure JaynesCummings evolution of the atom-plus-cavity systems takes a form that suggests stationarity, namely,

$$
\begin{aligned}
|e, n\rangle \rightarrow & \cos \left[\Omega \sqrt{n+1}\left(t_{1}-t_{2}\right)\right]|e, n\rangle \\
& +\sin \left[\Omega \sqrt{n+1}\left(t_{1}-t_{2}\right)\right]|g, n+1\rangle,
\end{aligned}
$$

$\Omega$ being the single-photon Rabi frequency.

Then, the probability for an atom to remain excited at time $t>0$ is given by

$$
Q_{e e}(t ; n)=\cos ^{2}[\Omega \sqrt{n+1} t]
$$

in the case of exact resonance.

Accordingly to the preceding section, a realist two-level stochastic process displays a temporal evolution where the conditional probabilities involved are restricted by two independent Bell-type inequalities. In order to fix a common range of forbidden values, let us rewrite (22) and (29) by introducing functions $B_{i}$, defined as

$$
\begin{gathered}
B_{1}(t)=Q_{e e}(0,2 t)+2 Q_{e e}(0, t)-2 \geqslant-1, \\
B_{2}(t)=Q_{e e}(0,2 t)-Q_{e e}^{2}(0, t)-1 \geqslant-1 .
\end{gathered}
$$

As clearly shown in Fig. 1, the periodic behavior exhibited by both $B_{1}$ and $B_{2}$ according to quantum mechanics leads to forbidden values for a wide range of interaction times. For instance, $B_{1}$ takes its most forbidden values for $\Omega t$ equal to $n \pi / 3, n$ being any integer that is not a multiple of 3 .

\section{B. A realistic experimental situation}

However, in a more realistic situation at a nonzero temperature, the cavity contains an indefinite number of photons and the Rabi solution (34) has to be averaged over the probability distribution $P_{n}$ of having initially $n$ photons in the mode, that is 


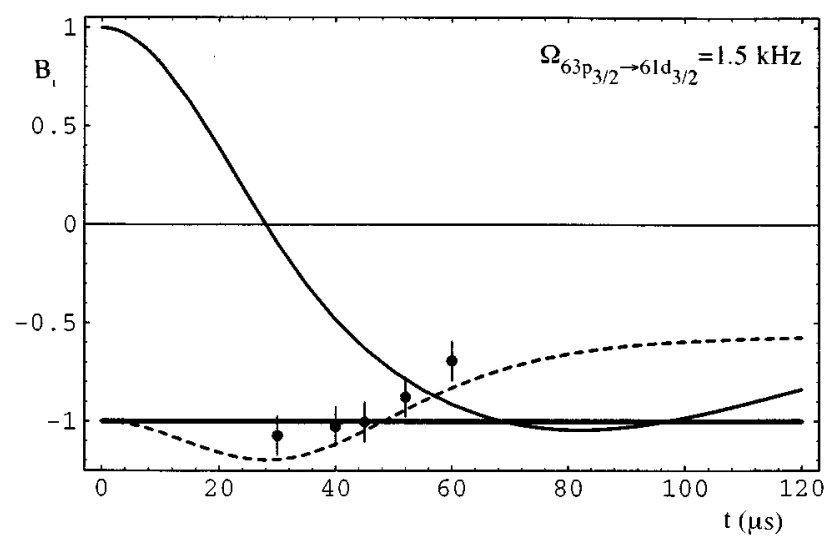

FIG. 2. $B_{1}$ and $B_{2}$ (dashed curve) vs $t$ in $\mu$ s at $T=3 \mathrm{~K}$. Violations of the Bell-type inequalities occur whenever either curve goes below -1 . We have represented the experimental data obtained in Ref. [26] corresponding to the transition $63 p_{3 / 2} \rightarrow 61 d_{3 / 2}(21506.5$ $\mathrm{MHz}$ ) of rubidium.

$$
Q_{e e}(t)=\sum_{n=0}^{\infty} P_{n} \cos ^{2}[\Omega \sqrt{n+1} t]
$$

In this case, the probabilities $P_{n}$ are easily calculated from Bose-Einstein statistics

$$
P_{n}=\frac{\bar{n}^{n}}{(\bar{n}+1)^{n+1}},
$$

$\bar{n}$ being the temperature-dependent mean photon number. In order to make a comparison with presently available experimental data, let us consider a setup analogous to that described in $[26,27]$. A velocity selected beam of rubidium atoms is prepared in the Rydberg level $63 P_{3 / 2}$ by laser excitation and passes through a superconducting closed microwave cavity, which can be tuned to two diferent Rydberg transitions $63 p_{3 / 2} \rightarrow 61 d_{3 / 2} \quad(21506.5 \quad \mathrm{MHz}) \quad$ and $63 p_{3 / 2} \rightarrow 61 d_{5 / 2}(21456.0 \mathrm{MHz})$. The corresponding Rabi frequencies have been estimated to be 1.5 and $7 \mathrm{kHz}$, respectively. The atoms exiting the cavity are detected in two separate field ionization detectors. The interaction time of an atom with the field can be varied by selecting different atomic velocities with a Fizeau filter.

For a temperature of $3 \mathrm{~K}$, the quality factor of the cavity is $Q=6 \times 10^{7}$. Under these conditions, the mean photon number is $\bar{n}=2.5$ with a cavity damping time of $500 \mu \mathrm{s}$. In addition to the requirement that $t_{\text {int }} \ll \tau$ for the strongcoupling regime, the atomic lifetime fixes the accessible interaction times in the range of $0-160 \mu \mathrm{s}$. Figure 2 shows the quantum-mechanical predictions for the functions $B_{i}$ in this domain; note that two measurements, at times $t$ and $2 t$, are required in order to obtain a single value of either $B_{i}$. On the other hand, the atomic flux has to be kept below $2000 \mathrm{~s}^{-1}$ in order that the cavity field relaxes back to thermal equilibrium between successive atoms. At $T=3 \mathrm{~K}$, only violations of the realistic bound for $B_{2}$ may be observed using the transition with smaller Rabi frequency. We have incorporated the experimental data presented in Ref. [26]. As can be seen in the figure, the statistics provided by Rempe et al. do not allow us to draw any conclusion. For instance, the pair of interaction

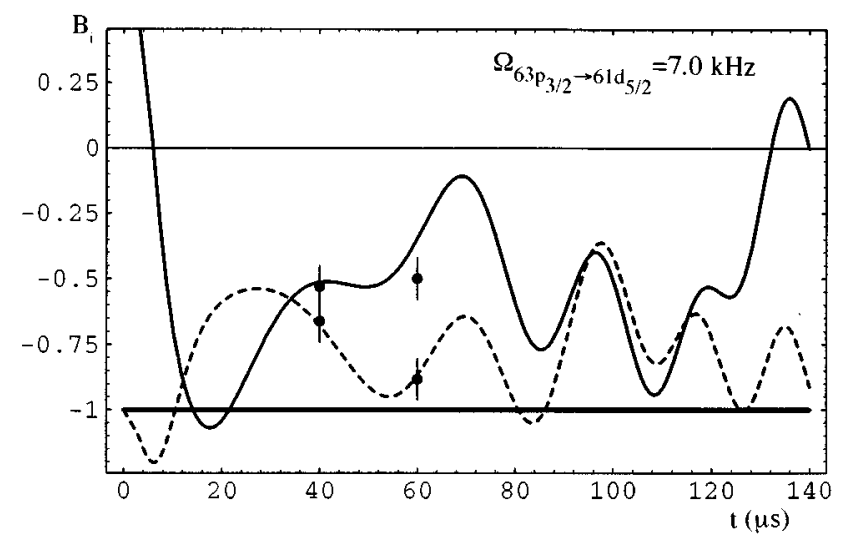

FIG. 3. $B_{1}$ and $B_{2}$ (dashed curve) vs $t$ in $\mu$ s at $T=2.5 \mathrm{~K}$. Violations of the Bell-type inequalities occur whenever either curve goes below -1 . We have represented the experimental data obtained in Ref. [26] for the transition $63 p_{3 / 2} \rightarrow 61 d_{5 / 2}(21456.0 \mathrm{MHz})$ of rubidium.

times of 30 and $60 \mu$ s yields a value of $B_{2}$ with a range of uncertainty between -0.96 and -1.19 .

At a temperature equal to $T=2 \mathrm{~K}$, the mean number of thermal photons decreases to $\bar{n}=2$ with a cavity damping time of $2 \mathrm{~ms}$ and a $Q$ factor of $2.7 \times 10^{8}$. We have shown in Fig. 3 the quantum mechanical prediction in this case, for the transition with larger Rabi frequency. Unfortunately, just a few measurements at short interaction times were performed (Fig. 3 in Ref. [25]) with an atomic flux suitable for our scheme to be applicable, and no conclusions can be drawn from those results.

In more recent experiments [28], the temperature is reduced to $0.5 \mathrm{~K}$ by means of a ${ }^{3} \mathrm{He}$ cryostat. Then $Q$ reaches the value of $3 \times 10^{10}$ and the storage time increases to $0.2 \mathrm{~s}$ with a mean photon number equal to 0.15 . In this case, the atomic flux has to be reduced below $5 \mathrm{~s}^{-1}$. No experiment performed to date satisfies this requirement.

As shown in Fig. 4, the decrease of $T$ results in an enhancement of the deviation from realistic bounds and the behavior of both $B_{i}$ differs only slightly from the situation in which the state of the cavity is described by a pure Fock

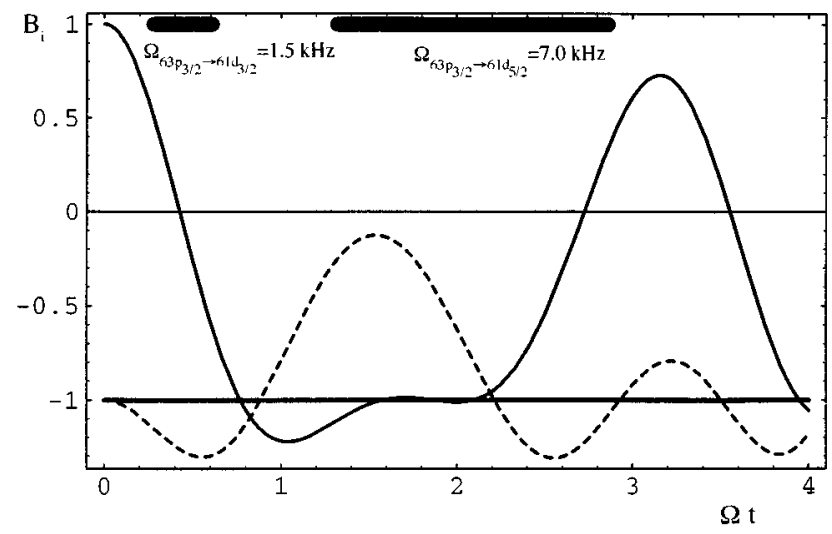

FIG. 4. $B_{1}$ and $B_{2}$ (dashed curve) vs $\Omega t$ at $T=0.5 \mathrm{~K}$. Violations of Bell-type inequalities occur whenever either curve goes below -1 . The thick horizontal segments indicate the ranges of interaction times accessible for the two Rydberg transitions. 


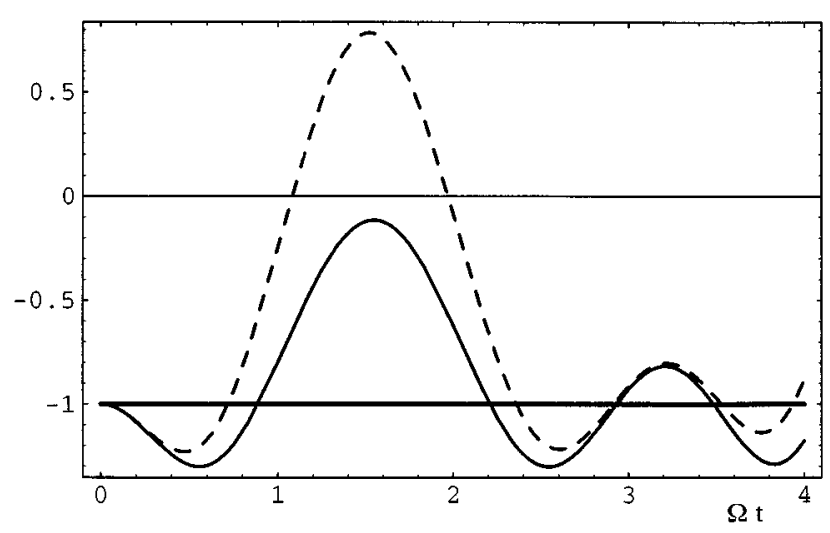

FIG. 5. $B_{2}$ clearly supersedes the function defined by the inequality (30), dashed curve in the figure. Note that $B_{2}$ covers the forbidden values attached by (30) and extends to wider regions. $T$ has been taken equal to $0.5 \mathrm{~K}$.

state, as in Fig. 1. Note that in the present case it might be possible to observe violations of both Bell-type inequalities using the transition with larger Rabi frequency. Finally, Fig. 5 shows the predicted behavior of $B_{2}$ compared to the function defined by the inequality (30). This shows the significance of the chain (31), since (22) clearly supersedes (30).

\section{Interaction with a coherent field}

In this case the probability distribution $P_{n}$ is Poissonian, that is

$$
P_{n}=\exp [-\bar{n}] \frac{\bar{n}^{n}}{n !} .
$$

The quantum-mechanical prediction for the functions $B_{i}$ has been plotted in Fig. 6 for $\bar{n}=5$. As expected, the characteristic behavior of an initial collapse followed by a nearly time independent evolution and then a revival displayed by the upper-level probability for the interaction of a two-level atom with a coherent field is exhibited by both functions $B_{i}$. At short interaction times, these functions yield forbid-

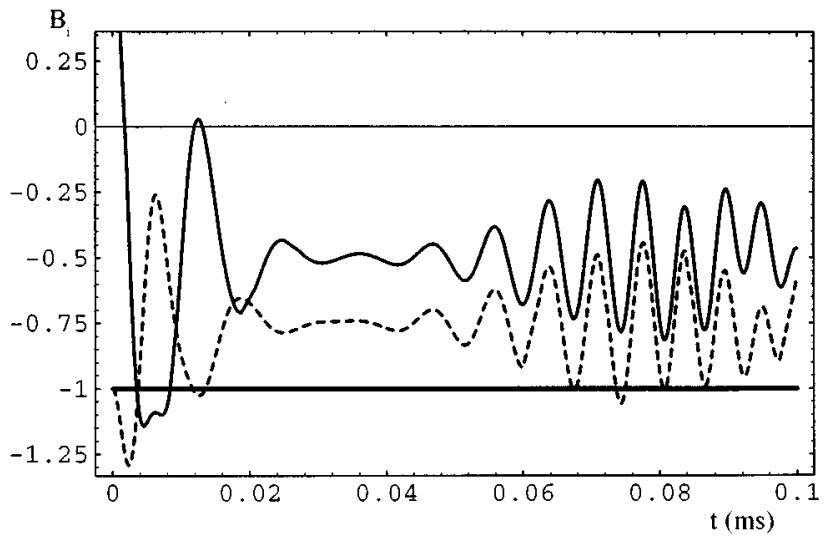

FIG. 6. $B_{1}$ and $B_{2}$ (dashed) for the interaction with a coherent field with $\bar{n}=5$. In addition to forbidden values at short interaction times exhibited in the thermal-field case, a second forbidden region apperars at long interaction times. It has been considered $\Omega=10^{5}$ $\mathrm{rad} / \mathrm{s}^{-1}$; the interaction time is written in milliseconds.

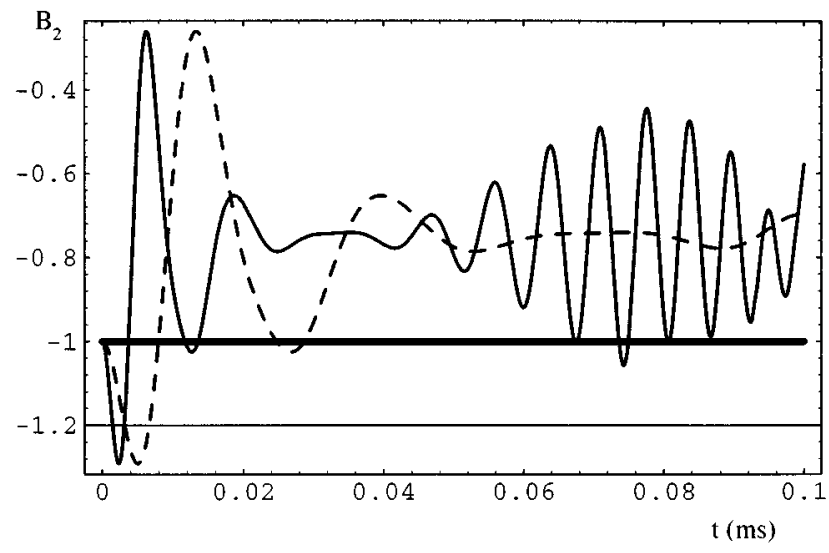

FIG. 7. Predicted behavior of function $B_{2}$ for elliptical (dashed curve) and circular Rydberg states of rubidium. The observation of forbidden values at long interaction times is unrealistic in the former case due to insufficiently longer atomic lifetime. Circular states exhibit larger values of both the coupling parameter $\Omega$ and $\tau_{\text {at }}$, hence being the ideal tools for the proposed test.

den values at different instants but for longer interaction times, only $B_{2}$ goes below the limit -1 , in the central region of the revival area. If such a violation of the Bell-type inequality (38) were observed, the revival predicted by the Jaynes-Cummings model could be classified as a purely quantum feature. As we shall show below, an experiment along these lines is feasible with present technology.

The preparation of the initial field presents no difficulty. A coherent field can be fed into the resonator by means of the coupling with a classical current source. The duration of the switching fixes the mean number of photons initially present in the cavity.

However, for long interactions, the setup of the preceding section can no longer be used to find departures from the realist bounds. This would require $\Omega t_{\mathrm{int}} \sim 8$, which gives $t_{\text {int }}=1.82 \times 10^{-4} \mathrm{~s}$ for the transition with $\Omega=7.5 \mathrm{kHz}$, and given that two measurements, at $t$ and $2 t$, are necessary in order to evaluate $B_{2}(t)$, we require an interaction time of the order of three times the atomic lifetime. This difficulty can be solved using circular Rydberg states, with maximum orbital and magnetic quantum numbers, $l=|m|=n-1$ (quasiclassical Bohr atom), see Fig. 7. The preparation of these states of rubidium and the efficiency of the procedure is described in [29]. Atoms prepared in circular states with principal quantum numbers $n \sim 50$ have a damping time $\tau_{\text {at }}$ of the order of $10 \mathrm{~ms}$, much longer than the interaction time of thermal atoms crossing a $\mathrm{cm}$-size resonator that our experiment requires. More concretely, the typical Rabi frequency between an $n=50$ circular atom and the cavity is $\Omega=10^{5}$ $\mathrm{rad} / \mathrm{s}^{-1}$, which gives, for instance, the pair of interaction times $t_{\text {int }}=\{0.075,0.150\} \mathrm{ms}$ in order for violations of the Bell-type inequality (38) to be observable in the long interaction time domain.

The use of circular states demands an open Fabry-Pérot type structure for the resonator. $Q$ factors as high as $3.10^{9}$ have been already achieved [30] using superconducting niobium cavities cooled at temperatures of the order of $1 \mathrm{~K}$. In this case, the cavity damping time is also of the order of 10 $\mathrm{ms}$ and hence, since $\tau_{\mathrm{at}}, \tau \gg t_{\text {int }}$, dissipative processes can be neglected. In order for the stationary assumption to be ad- 


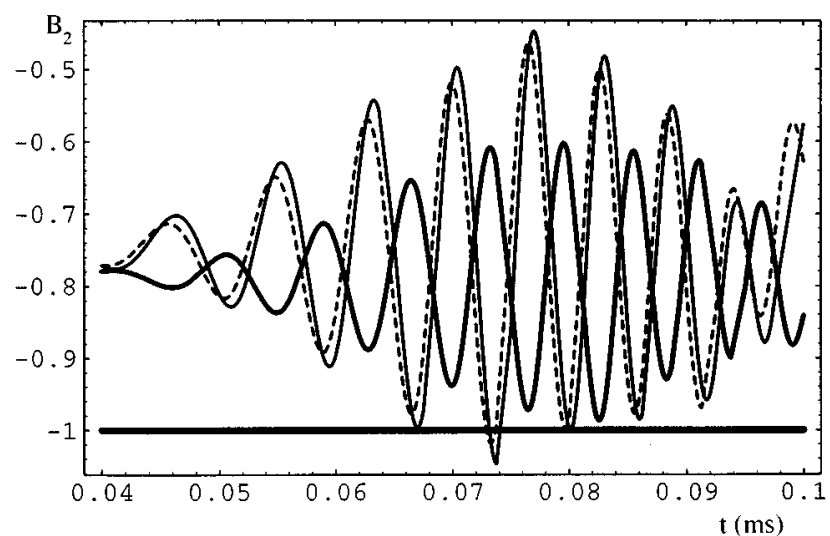

FIG. 8. Behavior of the function $B_{2}$ according to quantum mechanics for nonzero detuning. Solid line, $\delta=5 \mathrm{kHz}$; dashed line, $\delta=10 \mathrm{kHz}$; thick line, $\delta=25 \mathrm{kHz}$.

missible, the atomic flux cannot exceed $100 \mathrm{~s}^{-1}$.

As shown in Fig. 6, violations of the realistic limit in the revival region are small compared to those at very short interaction times. Hence the predicted effect will be very sensitive to all nonidealities present in a real experiment. In particular, the ideal condition of exact resonance has been considered so far. We have found that a maximum detuning of $10 \mathrm{kHz}$ is admissible for the function $B_{2}$ to exhibit values below -1 , as shown in Fig. 8.

The experiment seems also to be reliable with a cavity containing a coherent field with a somewhat higher mean photon number, between 10 and 15 . In that case there is not a great variation in the values of $B_{2}$, but there is a displacement to longer but still accessible interaction times, as can be seen in Fig. 9. However, from a conceptual point of view, there is no interest in increasing $\bar{n}$. Note that the state vector for the composite system involves, in each case, two terms differing by one photon, which is different from what happens in a genuine cat state, where two macroscopically distinct pure states of the field are involved. We will finish this section with two comments concerning the experimental realization of the proposal. The two measurements required for obtaining a single value of each function $B_{i}$ could be accomplished by successive measurements controlling the atomic

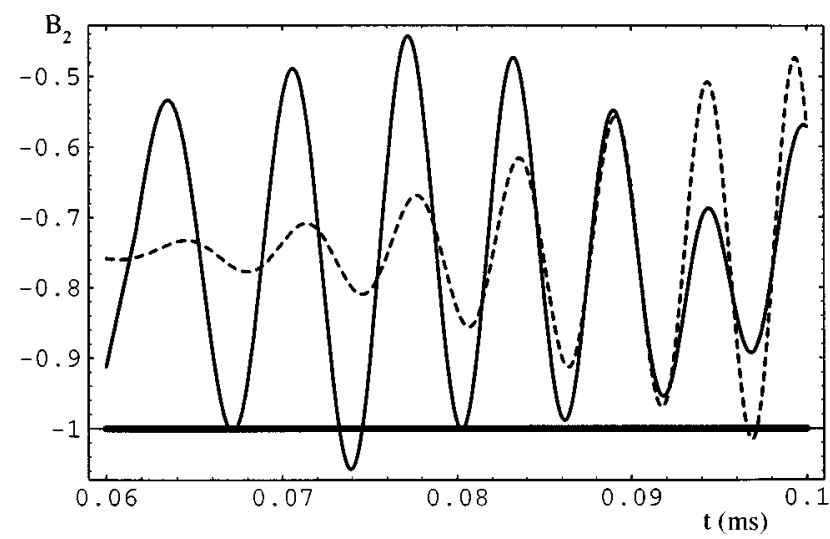

FIG. 9. Expected behavior of the function $B_{2}(t)$ in the long-time interaction domain for the interaction with a coherent field with mean photon number $\bar{n}=5$ and $\bar{n}=10$ (dashed curve). velocity in order to get the appropiate interaction times. Alternatively, the same velocity can be used for the two shots if a freezing electrode technique is employed for measuring the state of the atom after the time $t$. This technique consists of the application of a nonuniform electric field that Stark shifts the Rydberg levels out of resonance and then interrupts the coherent coupling between the atom and the cavity mode [31].

The second comment concerns the atomic detectors. It should be noted that, unless one deals with perfect detectors, the measurement of the conditional probabilities $Q_{e e}$ requires that we determine the number of atoms, $N_{e}$ and $N_{g}$, exiting the cavity in states $e$ and $g$ after the interaction time $t, N_{e}$. Then, if both channeltrons have the same efficiency, a sequence of such measurements would simply provide a scaled curve that could be identified with the absolute one if a faithful sampling assumption is made, that is,

$$
\left.Q_{e e}(0, t)\right|_{\text {expt }}=\frac{N_{e}}{N_{e}+N_{g}} .
$$

This method is not applicable, however, if the field ionization detectors have different efficiency. In this case the corresponding efficiencies have to be determined by an independent procedure.

\section{SUMMARY AND CONCLUSIONS}

As pointed out in various parts of this paper, our experimental proposal addresses the Schrödinger cat problem at a level that can be considered as intermediate between the microscopic and the macroscopic, in the sense that a quasiclassical two-level Rydberg atom coupled to a single mode with an indefinite number of photons may be expected to exhibit certain classical properties. Note "mesoscopic" in cavity QED often has a different meaning. It refers to situations where a few tenths of atoms and/or photons are involved. Here the word mesoscopic is applied only to the linear dimensions of the atomic system.

On this basis, we have developed arguments for the temporal evolution of the composite system to be described by a two-level stationary stochastic process. This allows us to derive two independent temporal Bell-type inequalities to be fulfilled according to the realist description, and directly testable by measuring the number of atoms exiting the cavity in the two different atomic states, $e$ or $g$, after a controlled interaction time with the cavity mode. As shown in Sec. III, the quantum mechanical predictions violate the realist bounds in different ranges depending on the initial field statistics. In order to observe such violations in a real experiment, we propose two different tests in which the cavity contains initially a thermal field at very low temperature, and a coherent field. The first type of test can be accomplished with a setup analogous to that decribed by Rempe et al. $[26,27]$. Using the transition with larger $\Omega$ and at a temperature equal to $0.5 \mathrm{~K}$, violations of both Bell-type inequalities should be observable with interaction times ranging from 20 to $60 \mu \mathrm{s}$. The second type of experiment exhibits a different profile where two separate forbidden regions appear. At very short interaction times, below $10 \mu \mathrm{s}$, both functions $B_{i}$ go below the realist limit -1 . Such a violation could be ob- 
served with the same setup as before. However, violations at longer interaction times demand the use of long-lived circular Rydberg states and hence open structures as resonators with a quality factor of the order of $10^{9}$. In that case, the central area of the revival region yields forbidden values. This fact throws new light on the quantum nature of the so-called revival dynamics in contrast with classical behavior. The interaction times required for its observation range from 73 to $75 \mu \mathrm{s}$. It should be stressed that, in order to determine a single value of each function $B_{i}$, two measurents, at times $t$ and $2 t$, are required. We conclude that a violation of these Bell-type inequalities, in addition to testing the validity of the quantum formalism at scales where some classical properties emerge, would clarify the requirements for a realist theory to reproduce the experimental data. As realism alone does not constitute a source of incompatibility with the quantum mechanical predictions, the additional assumption(s) introduced in order to derive testable inequali- ties would not hold. Then, an empirical violation of the derived Bell-type inequalities would indicate that any hidden variables interpretation of the experiment in terms of just two states is untenable. In other words, if such an interpretation is possible at all, the model would have to incorporate additional states to account for the fact that when an atom, initially in state $e$, is detected in state $e$ after interaction times $t$ and $2 t$, the state of the cavity field is not necessarily the same, nor is either the same as the field initially encountered. The formal role of these states in the model would be analogous to the superposition states in the quantum description.

\section{ACKNOWLEDGMENTS}

We are grateful to J. M. Raimond and J. I. Cirac for some useful comments concerning circular Rydberg states. This research work was supported by DGICYT Project No. PB92-0507 (Spain).
[1] S. F. Huelga, T. W. Marshall, and E. Santos, Phys. Rev. A 52, R2497 (1995).

[2] A. Einstein, B. Podolsky, and N. Rosen, Phys. Rev. 47, 777 (1935).

[3] E. Schrödinger, Naturwissenschaften 23, 807 (1935).

[4] J. S. Bell, Physica 1, 195 (1964); Rev. Mod. Phys. 38, 447 (1966).

[5] Quantum Mechanics versus Local Realism, edited by F. Seller (Plenum, New York, 1988).

[6] L. Mandel, Ann. N.Y. Acad. Sci. 755, 1 (1995), and references therein.

[7] E. Santos, Phys. Rev. Lett. 66, 1388 (1991); 68, 2702 (1992); Phys. Rev. A 46, 3646 (1992).

[8] S. J. D. Phoenix and S. M. Barnett, J. Mod. Opt. 40, 979 (1993).

[9] J. I. Cirac and P. Zoller, Phys. Rev. A 50, R2799 (1994).

[10] D. M. Greenberger, M. A. Horne, and A. Zeilinger, in Bell's Theorem, Quantum Theory and Conceptions of the Universe, edited by M. Kafatos (Kluwer, Dordrecht, 1989).

[11] A. J. Leggett and A. Garg, Phys. Rev. Lett. 54, 587 (1985).

[12] C. D. Tesche, Phys. Rev. Lett. 64, 2358 (1990).

[13] J. P. Paz and G. Mahler, Phys. Rev. Lett. 71, 3235 (1993).

[14] Proceedings of the International Conference on Mesoscopic Physics and Fundamental Problems in Quantum Mechanics, Rome, June 1994 [Nuovo Cimento 110B (1995)].

[15] S. Haroche, M. Brune, J. M. Raimond, and L. Davidovich, in Fundamentals of Quantum Optics III (Springer Verlag, New York, 1993).
[16] J. S. Bell, in Speakable and Unpeakable in Quantum Mechanics (Cambridge University, Cambridge, 1987), p. 89.

[17] M. Ferrero, T. W. Marshall, and E. Santos, Am. J. Phys. 58, 683 (1990).

[18] E. S. Fry, T. Walther, and S. Li, Phys. Rev. A 52, 4381 (1995).

[19] P. G. Kwiat et al., Phys. Rev. A 49, 3209 (1994).

[20] S. F. Huelga, M. Ferrero, and E. Santos, Europhys. Lett. 27, 181 (1994); Phys. Rev. A 51, 5008 (1995)

[21] W. H. Zurek, Phys. Today 44 (10), 36 (1992).

[22] J. F. Clauser et al., Phys. Rev. Lett. 23, 880 (1969).

[23] L. Allen and J. H. Eberly, Optical Resonance and Two Level Atoms (Wiley, New York, 1975), p. 159.

[24] B. Shore and P. L. Knight, J. Mod. Opt. 40, 1195 (1993).

[25] P. Filipowicz, J. Javanainen, and P. Meystre, Phys. Rev. A 34, 3007 (1986).

[26] G. Rempe, H. Walther, and N. Klein, Phys. Rev. Lett. 58, 353 (1987).

[27] G. Rempe, F. Schmidt-Kaler, and H. Walther, Phys. Rev. Lett. 64, 2783 (1990).

[28] H. Walther, Phys. Rep. 219, 263 (1992).

[29] J. P. Nussenzweig et al., Phys. Rev. Lett. 48, 3991 (1993).

[30] J. M. Raimond and S. Haroche, in Quantum Fluctuations, Les Houches Session LXIII, edited by E. Giacobino, S. Reynaud, and J. Zinn-Justin (North-Holland, Amsterdam, in press).

[31] S. Haroche, in New Trends in Atomic Physics, Les Houches: Section XXXVIII. Vol. 1, edited by G. Grynberg and R. Stora (North-Holland, Amsterdam, 1982), pp. 212-213. 